\title{
MEASURE THEORY AND INTEGRATION ON THE LEVI-CIVITA FIELD
}

\author{
KHODR SHAMSEDDINE AND MARTIN BERZ
}

\begin{abstract}
It is well known that the disconnectedness of a non-Archimedean totally ordered field in the order topology makes integration more difficult than in the real case. In this paper, we present a remedy to that difficulty and study measure theory and integration on the Levi-Civita field. After reviewing basic elements of calculus on the field, we introduce a measure that proves to be a natural generalization of the Lebesgue measure on the field of the real numbers and have similar properties. Then we introduce a family of simple functions from which we obtain a larger family of measurable functions and derive a simple characterization of such functions. We study the properties of measurable functions, we show how to integrate them over measurable sets of $\mathcal{R}$, and we show that the resulting integral satisfies similar properties to those of the Lebesgue integral of real calculus.
\end{abstract}

\section{INTRODUCTION}

Measure theory and integration on the Levi-Civita field $\mathcal{R}[5,6]$ are presented. We start with a review of some basic and useful terminology and refer the reader to $[1,10,2,11]$ for a more detailed study of the Levi-Civita field. For a general overview of the algebraic properties of formal power series fields in general, we refer to the comprehensive overview by Ribenboim [9], and for an overview of the related valuation theory the book by Krull [3]. A thorough and complete treatment of ordered structures can also be found in [8].

Definition 1.1 (The set $\mathcal{R}$ ). We define $\mathcal{R}=\{f: \mathbb{Q} \rightarrow \mathbb{R}:\{x \in \mathbb{Q}: f(x) \neq 0\}$ is left-finite $\}$. So the elements of $\mathcal{R}$ are those real valued functions on $\mathbb{Q}$ that are

1991 Mathematics Subject Classification. 11S80, 12J25, 26E30, 46S10.

Key words and phrases. Levi-Civita field, non-Archimedean calculus, measurable sets, measurable functions, integration.

This research was supported by an Alfred P. Sloan fellowship and by the United States Department of Energy, Grant \# DE-FG02-95ER40931.

(C) 2003 American Mathematical Society 
non-zero only on a left-finite set, i.e. below every rational number $q$ there are only finitely many points where the given functions do not vanish.

We denote elements of $\mathcal{R}$ by $x, y$, etc. and identify their values at $q \in \mathbb{Q}$ with brackets like $x[q]$. This avoids confusion when we consider functions on $\mathcal{R}$. For the further discussion, it is convenient to introduce the following terminology.

Definition 1.2 (supp, $\lambda, \sim, \approx,=_{r}$ ). For $x \in \mathcal{R}$, we denote $\{q \in \mathbb{Q}: x[q] \neq 0\}$ by $\operatorname{supp}(x)$ and call it the support of $x$; and we define $\lambda(x)=\min (\operatorname{supp}(x))$ for $x \neq 0$ (which exists because of left-finiteness), and $\lambda(0)=+\infty$.

Comparing two elements $x$ and $y$ in $\mathcal{R}$, we say $x \sim y$ if $\lambda(x)=\lambda(y), x \approx y$ if $\lambda(x)=\lambda(y)$ and $x[\lambda(x)]=y[\lambda(y)]$, and $x={ }_{r} y$ if $x[q]=y[q]$ for all $q \leq r$.

At this point, these definitions may feel somewhat arbitrary; but after having introduced the concept of ordering on $\mathcal{R}$, we will see that $\lambda$ describes "orders of infinite largeness or smallness", the relation " $\approx$ " corresponds to agreement up to infinitely small relative error, while " $"$ corresponds to agreement of order of magnitude.

Definition 1.3 (Addition and Multiplication on $\mathcal{R}$ ). We define addition on $\mathcal{R}$ componentwise: $(x+y)[q]=x[q]+y[q]$. Multiplication is defined as follows: For $q \in Q$, we set $(x \cdot y)[q]=\sum_{q_{x}+q_{y}=q} x\left[q_{x}\right] \cdot y\left[q_{y}\right]$.

Since elements of $\mathcal{R}$ have left-finite supports, only finitely many terms contribute to the sum in the definition of multiplication. Thus, $\cdot$ is a well defined operation on $\mathcal{R}$. It turns out that the operations + and $\cdot$ make $(\mathcal{R},+, \cdot)$ into a field, in which we can isomorphically embed $\mathbb{R}$ as a subfield via the map $\Pi: \mathbb{R} \rightarrow \mathcal{R}$ defined by

$$
\Pi(x)[q]= \begin{cases}x & \text { if } q=0 \\ 0 & \text { otherwise }\end{cases}
$$

Definition 1.4 (Ordering in $\mathcal{R}$ ). Let $x, y$ be distinct elements of $\mathcal{R}$. We say $x>y$ if $(x-y)[\lambda(x-y)]>0$. Furthermore, we say $x<y$ if $y>x$.

With this definition of the order relation, $\mathcal{R}$ is a totally ordered field. Moreover, the embedding $\Pi$ in (1.1) is compatible with the order. Besides the usual order relations, some other notations are also convenient.

Definition 1.5. ( $\ll, \gg)$ Let $a, b$ be non-negative. We say $a$ is infinitely smaller than $b$ (and write $a \ll b$ ) if $n \cdot a<b$ for all natural $n$; we say $a$ is infinitely larger than $b$ (and write $a \gg b$ ) if $b \ll a$. If $a \ll 1$, we say $a$ is infinitely small; if $1 \ll a$, we say $a$ is infinitely large. Non-negative numbers that are neither infinitely small nor infinitely large are also called finite.

Definition 1.6 (The Number $d$ ). Let $d$ be the element of $\mathcal{R}$ given by $d[1]=1$ and $d[q]=0$ for $q \neq 1$.

It is easy to check that $0<d \ll 1$. It follows that, altogether, the Levi-Civita $\mathcal{R}$ is a totally ordered non-Archimedean field extension of the real numbers. It is shown [1] that this field is totally disconnected in the natural topology induced by the order. Because of this disconnectedness, which translates into the existence of an enormous number of "holes" between the different orders, suprema and infima even of bounded sets do not always exist. Moreover, there are nonconstant differentiable functions with vanishing derivatives everywhere on $\mathcal{R}$; consequently, for a given 
function there could exist multiple anti-derivatives. Thus, trying to extend the Riemann integral or the Lebesgue integral from $\mathbb{R}$ to $\mathcal{R}$ is not all straightforward.

In this paper, we successfully circumvent the difficulties mentioned above and define a measure on the Levi-Civita field $\mathcal{R}$ that we prove is a natural generalization of the Lebesgue measure on $\mathbb{R}$ and has similar properties. Namely, we show that any subset of a measurable set of measure 0 is itself measurable and has measure 0 . We also show that any countable unions of measurable sets whose measures form a null sequence is measurable and the measure of the union is less than or equal to the sum of the measures of the original sets; moreover, the measure of the union is equal to the sum of the measures of the original sets if the latter are mutually disjoint. Then we show that any finite intersection of measurable sets is also measurable and that the sum of the measures of two measurable sets is equal to the sum of the measures of their union and intersection.

We introduce the concept of measurable functions on measurable sets of $\mathcal{R}$ through a smaller family of simple functions, we derive a simple characterization of such functions and we show that they form an algebra. Then we show that a measurable function is differentiable almost everywhere and that a function measurable on two measurable subsets of $\mathcal{R}$ is also measurable on their union and intersection.

We define the integral of a measurable function $f$ over a measurable set $A$ and show that the integral satisfies similar properties to those of the Lebesgue and Riemann integrals on $\mathbb{R}$. In particular, we prove the linearity property of the integral and that if $|f| \leq M$ on $A$ then $\left|\int_{A} f\right| \leq M m(A)$, where $m(A)$ is the measure of $A$. We also show that the sum of the integrals of a measurable function over two measurable sets is equal to the sum of its integrals over the union and the intersection of the two sets. Finally, we show that if $\left(f_{n}\right)$ is a sequence of measurable functions on a measurable set $A$ that converges uniformly on $A$, then the integrals of $f_{n}$ over $A$ form a converging sequence in $\mathcal{R}$. Moreover, if the uniform limit $f$ of the functions $f_{n}$ is itself measurable on $A$, then its integral $\int_{A} f$ is equal to the limit of $\int_{A} f_{n}$.

\section{Measurable Sets}

Before we define a measure on $\mathcal{R}$, we introduce the following notations which will be adopted throughout this paper: $I(a, b)$ will be used to denote any one of the intervals $[a, b],(a, b],[a, b)$ or $(a, b)$, unless we explicitly specify a particular choice of one of the four intervals. Also, to denote the length of a given interval $I$, we will use the notation $l(I)$.

Definition 2.1. Let $A \subset \mathcal{R}$ be given. Then we say that $A$ is measurable if for every $\epsilon>0$ in $\mathcal{R}$, there exist a sequence of mutually disjoint intervals $\left(I_{n}\right)$ and a sequence of mutually disjoint intervals $\left(J_{n}\right)$ such that $\cup_{n=1}^{\infty} I_{n} \subset A \subset \cup_{n=1}^{\infty} J_{n}$, $\sum_{n=1}^{\infty} l\left(I_{n}\right)$ and $\sum_{n=1}^{\infty} l\left(J_{n}\right)$ converge in $\mathcal{R}$, and $\sum_{n=1}^{\infty} l\left(J_{n}\right)-\sum_{n=1}^{\infty} l\left(I_{n}\right) \leq \epsilon$.

Given a measurable set $A$, then for every $k \in \mathbb{N}$, we can select a sequence of mutually disjoint intervals $\left(I_{n}^{k}\right)$ and a sequence of mutually disjoint intervals $\left(J_{n}^{k}\right)$ such that $\sum_{n=1}^{\infty} l\left(I_{n}^{k}\right)$ and $\sum_{n=1}^{\infty} l\left(J_{n}^{k}\right)$ converge in $\mathcal{R}$ for all $k$,

$$
\cup_{n=1}^{\infty} I_{n}^{k} \subset \cup_{n=1}^{\infty} I_{n}^{k+1} \subset A \subset \cup_{n=1}^{\infty} J_{n}^{k+1} \subset \cup_{n=1}^{\infty} J_{n}^{k} \text { and } \sum_{n=1}^{\infty} l\left(J_{n}^{k}\right)-\sum_{n=1}^{\infty} l\left(I_{n}^{k}\right) \leq d^{k}
$$


for all $k \in \mathbb{N}$. Since $\mathcal{R}$ is Cauchy-complete in the order topology, it follows that $\lim _{k \rightarrow \infty} \sum_{n=1}^{\infty} l\left(I_{n}^{k}\right)$ and $\lim _{k \rightarrow \infty} \sum_{n=1}^{\infty} l\left(J_{n}^{k}\right)$ both exist and they are equal. We call the common value of the limits the measure of $A$ and we denote it by $m(A)$. Thus,

$$
m(A)=\lim _{k \rightarrow \infty} \sum_{n=1}^{\infty} l\left(I_{n}^{k}\right)=\lim _{k \rightarrow \infty} \sum_{n=1}^{\infty} l\left(J_{n}^{k}\right) .
$$

Moreover, since the sequence $\left(\sum_{n=1}^{\infty} l\left(I_{n}^{k}\right)\right)_{k \in \mathbb{N}}$ is nondecreasing and since the sequence $\left(\sum_{n=1}^{\infty} l\left(J_{n}^{k}\right)\right)_{k \in \mathrm{N}}$ is nonincreasing, we have that

$$
\sum_{n=1}^{\infty} l\left(I_{n}^{k}\right) \leq m(A) \leq \sum_{n=1}^{\infty} l\left(J_{n}^{k}\right) \text { for all } k \in \mathbb{N} .
$$

Contrary to the real case, $\sup \left\{\sum_{n=1}^{\infty} l\left(I_{n}\right): I_{n}\right.$ 's are mutually disjoint intervals and $\left.\cup_{n=1}^{\infty} I_{n} \subset A\right\}$ and $\inf \left\{\sum_{n=1}^{\infty} l\left(J_{n}\right): A \subset \cup_{n=1}^{\infty} J_{n}\right\}$ need not exist for a given set $A \subset \mathcal{R}$. However, as we will show in Proposition 2.2, if $A$ is measurable then both the supremum and infimum exist and they are equal to $m(A)$. This shows that the definition of measurable sets in Definition 2.1 is a natural generalization of the Lebesgue measure of real analysis that corrects for the lack of suprema and infima in non-Archimedean totally ordered fields.

Proposition 2.2. Let $A \subset \mathcal{R}$ be measurable. Then

$$
\begin{aligned}
& m(A) \\
= & \inf \left\{\sum_{n=1}^{\infty} l\left(J_{n}\right): J_{n} \text { is an interval } \forall n, A \subset \cup_{n=1}^{\infty} J_{n} \text { and } \sum_{n=1}^{\infty} l\left(J_{n}\right) \text { converges }\right\} \\
= & \sup \left\{\sum_{n=1}^{\infty} l\left(I_{n}\right): I_{n} \text { 's are mutually disjoint, } \cup_{n=1}^{\infty} I_{n} \subset A, \sum_{n=1}^{\infty} l\left(I_{n}\right) \text { converges }\right\} .
\end{aligned}
$$

Proof. First we show that the infimum exists and is equal to $m(A)$. Using Equation (2.1) and Equation (2.2), it remains to show that if $\left(J_{n}\right)$ is a sequence of intervals such that $\sum_{n=1}^{\infty} l\left(J_{n}\right)$ converges and $A \subset \cup_{n=1}^{\infty} J_{n}$ then $m(A) \leq \sum_{n=1}^{\infty} l\left(J_{n}\right)$. Assume not, then there exists a sequence of intervals $\left(J_{n}^{0}\right)$ such that $\sum_{n=1}^{\infty} l\left(J_{n}^{0}\right)$ converges, $A \subset \cup_{n=1}^{\infty} J_{n}^{0}$, but $m(A)>\sum_{n=1}^{\infty} l\left(J_{n}^{0}\right)$. Let $k \in \mathbb{N}$ be such that

$$
d^{k}<\frac{m(A)-\sum_{n=1}^{\infty} l\left(J_{n}^{0}\right)}{2}
$$

and let $\left(I_{n}^{k}\right)$ and $\left(J_{n}^{k}\right)$ be as in the discussion leading to Equation (2.1) and Equation (2.2). Then $\cup_{n=1}^{\infty} I_{n}^{k} \subset A \subset \cup_{n=1}^{\infty} J_{n}^{k}$ and $\sum_{n=1}^{\infty} l\left(J_{n}^{k}\right)-\sum_{n=1}^{\infty} l\left(I_{n}^{k}\right) \leq d^{k}$. Since $\cup_{n=1}^{\infty} I_{n}^{k} \subset A \subset \cup_{n=1}^{\infty} J_{n}^{0}$ and since the intervals $I_{n}^{k}$ are mutually disjoint, we obtain that

$$
\sum_{n=1}^{\infty} l\left(J_{n}^{0}\right) \geq \sum_{n=1}^{\infty} l\left(I_{n}^{k}\right)
$$

On the other hand, it follows from Equation (2.2) that

$$
m(A)-\sum_{n=1}^{\infty} l\left(I_{n}^{k}\right) \leq \sum_{n=1}^{\infty} l\left(J_{n}^{k}\right)-\sum_{n=1}^{\infty} l\left(I_{n}^{k}\right) \leq d^{k}
$$


Thus,

$$
\begin{aligned}
\sum_{n=1}^{\infty} l\left(J_{n}^{0}\right)-\sum_{n=1}^{\infty} l\left(I_{n}^{k}\right) & =\left(\sum_{n=1}^{\infty} l\left(J_{n}^{0}\right)-m(A)\right)+\left(m(A)-\sum_{n=1}^{\infty} l\left(I_{n}^{k}\right)\right) \\
& \leq\left(\sum_{n=1}^{\infty} l\left(J_{n}^{0}\right)-m(A)\right)+d^{k} \\
& <\left(\sum_{n=1}^{\infty} l\left(J_{n}^{0}\right)-m(A)\right)+\frac{m(A)-\sum_{n=1}^{\infty} l\left(J_{n}^{0}\right)}{2} \\
& =\frac{\sum_{n=1}^{\infty} l\left(J_{n}^{0}\right)-m(A)}{2} \\
& <0,
\end{aligned}
$$

which contradicts Equation (2.3).

Similarly, we show that $\sup \left\{\sum_{n=1}^{\infty} l\left(I_{n}\right): I_{n}\right.$ is an interval for each $n, I_{n}$ 's are mutually disjoint, $\cup_{n=1}^{\infty} I_{n} \subset A$ and $\sum_{n=1}^{\infty} l\left(I_{n}\right)$ converges $\}$ exists and is equal to $m(A)$.

It follows directly from Definition 2.1 and Proposition 2.2 that $m(A) \geq 0$ for any measurable set $A \subset \mathcal{R}$ and that any interval $I(a, b)$ is measurable with measure $m(I(a, b))=b-a$. It also follows that if $A$ is a countable union of mutually disjoint intervals $\left(I_{n}\left(a_{n}, b_{n}\right)\right)$ such that $\sum_{n=1}^{\infty}\left(b_{n}-a_{n}\right)$ converges then $A$ is measurable with $m(A)=\sum_{n=1}^{\infty}\left(b_{n}-a_{n}\right)$. Moreover, if $B \subset A \subset \mathcal{R}$ and if $A$ and $B$ are measurable, then $m(B) \leq m(A)$.

Proposition 2.3. Let $A \subset \mathcal{R}$ be measurable with $m(A)=0$ and let $B \subset A$. Then $B$ is measurable and $m(B)=0$.

Proof. First we show that $B$ is measurable. Let $\epsilon>0$ in $\mathcal{R}$ be given. By Proposition 2.2 , there exists a sequence of mutually disjoint intervals $\left(J_{n}\right)$ such that $A \subset$ $\cup_{n=1}^{\infty} J_{n}, \sum_{n=1}^{\infty} l\left(J_{n}\right)$ converges in $\mathcal{R}$, and $\sum_{n=1}^{\infty} l\left(J_{n}\right) \leq \epsilon$. For each $n \in \mathbb{N}$, let $I_{n}=$ $\emptyset$. Then $\cup_{n=1}^{\infty} I_{n} \subset B \subset \cup_{n=1}^{\infty} J_{n}$ and $\sum_{n=1}^{\infty} l\left(J_{n}\right)-\sum_{n=1}^{\infty} l\left(I_{n}\right)=\sum_{n=1}^{\infty} l\left(J_{n}\right) \leq \epsilon$. Hence $B$ is measurable. Since $B \subset A$, we obtain that $0 \leq m(B) \leq m(A)=0$. Thus, $m(B)=0$.

Proposition 2.4. Let $A \subset \mathcal{R}$ be countable. Then $A$ is measurable and $m(A)=0$.

Proof. Since $A$ is countable, we may write $A=\left\{a_{n}: n \in \mathbb{N}\right\}$. Now let $\epsilon>0$ be given in $\mathcal{R}$; for each $n \in \mathbb{N}$, let $J_{n}^{0}=\left(a_{n}-d^{n} \epsilon, a_{n}+d^{n} \epsilon\right)$ and $J_{n}=J_{n}^{0} \backslash \cup_{i=1}^{n-1} J_{i}^{0}$. Then $\cup_{n=1}^{\infty} J_{n}$ is a countable union of mutually disjoint intervals which we can write as $K_{1}, K_{2}, \ldots$, where $K_{1}=J_{1}=J_{1}^{0}, K_{2}$ and $K_{3}$ are the two mutually disjoint intervals in $J_{2}=J_{2}^{0} \backslash J_{1}^{0}$, and so on. Since $\cup_{i=1}^{\infty} K_{i}=\cup_{n=1}^{\infty} J_{n}=\cup_{n=1}^{\infty} J_{n}^{0}$, we obtain that $A \subset \cup_{i=1}^{\infty} K_{i}$. Also, since $\lim _{n \rightarrow \infty} l\left(J_{n}^{0}\right)=\lim _{n \rightarrow \infty} 2 d^{n} \epsilon=0$, it follows that $\lim _{i \rightarrow \infty} l\left(K_{i}\right)=0$. Hence $\sum_{i=1}^{\infty} l\left(K_{i}\right)$ converges in $\mathcal{R}[11]$ and

$$
\sum_{i=1}^{\infty} l\left(K_{i}\right) \leq \sum_{n=1}^{\infty} l\left(J_{n}^{0}\right)=\sum_{n=1}^{\infty} 2 d^{n} \epsilon=\frac{2 d}{1-d} \epsilon<\epsilon .
$$

Now for all $i \in \mathbb{N}$, let $I_{i}=\emptyset$. Then $\cup_{i=1}^{\infty} I_{i} \subset A \subset \cup_{i=1}^{\infty} K_{i}$ and $\sum_{i=1}^{\infty} l\left(K_{i}\right)-$ $\sum_{i=1}^{\infty} l\left(I_{i}\right)=\sum_{i=1}^{\infty} l\left(K_{i}\right)<\epsilon$. Thus, $A$ is measurable. Moreover, by Proposition 2.2 , we have that $m(A) \leq \sum_{i=1}^{\infty} l\left(K_{i}\right)<\epsilon$. This is true for all $\epsilon>0$ in $\mathcal{R}$; and hence $m(A)=0$. 
The converse of Proposition 2.4 is not necessarily true, that is if $A$ is measurable and if $m(A)=0$ then $A$ need not be countable, as the following example shows.

Example 2.5 (Cantor-like Set). A Cantor-like set $C_{\mathcal{R}}$ is constructed in the same way as the standard real Cantor set $C$; but instead of deleting the middle third, we delete the middle $(1-2 d)$ th of each of the closed subintervals of $[0,1]$ at each step of the construction. So first, we delete the interval $(d, 1-d)$ from the interval $F_{0}=[0,1]$ and let $F_{1}$ denote the remaining closed set consisting of the two closed intervals $[0, d]$ and $[1-d, 1]$ of length $d$ each. Then we delete the open intervals $\left(d^{2}, d-d^{2}\right)$ and $\left(1-d+d^{2}, 1-d^{2}\right)$, and let $F_{2}$ denote the remaining closed set which consists of four closed intervals $\left[0, d^{2}\right],\left[d-d^{2}, d\right],\left[1-d, 1-d+d^{2}\right]$ and $\left[1-d^{2}, 1\right]$ of length $d^{2}$ each. Then we delete the middle $(1-2 d)$ th from each of these four intervals, getting a new closed set $F_{3}$, consisting of eight closed intervals of length $d^{3}$ each, and so on. Continuing this process indefinitely (as we do in the construction of the real Cantor set $C$ ), we get a sequence of closed sets $F_{n}$ such that $F_{n} \subset F_{n-1}$ for all $n \in \mathbb{N}$. The intersection $C_{\mathcal{R}}=\cap_{n=0}^{\infty} F_{n}$ will be called the Cantor-like set.

Clearly, $C_{\mathcal{R}}$ is closed and uncountable since there is a one-to-one correspondence between $C$ and $C_{\mathcal{R}}$. We leave it as an exercise for the reader to show that while $C$ is not measurable in the context of the measure defined here on $\mathcal{R}, C_{\mathcal{R}}$ is measurable and $m\left(C_{\mathcal{R}}\right)=0$.

Proposition 2.6. For each $k \in \mathbb{N}$, let $A_{k} \subset \mathcal{R}$ be measurable such that $\left(m\left(A_{k}\right)\right)$ forms a null sequence. Then $\cup_{k=1}^{\infty} A_{k}$ is measurable and

$$
m\left(\cup_{k=1}^{\infty} A_{k}\right) \leq \sum_{k=1}^{\infty} m\left(A_{k}\right) .
$$

Moreover, if the sets $\left(A_{k}\right)_{k=1}^{\infty}$ are mutually disjoint, then

$$
m\left(\cup_{k=1}^{\infty} A_{k}\right)=\sum_{k=1}^{\infty} m\left(A_{k}\right) .
$$

Proof. First we note that, since $\lim _{k \rightarrow \infty} m\left(A_{k}\right)=0$, the sum $\sum_{k=1}^{\infty} m\left(A_{k}\right)$ converges [11]. Now let $\epsilon>0$ in $\mathcal{R}$ be given. Then for each $k \in \mathbb{N}$ there exist a sequence of mutually disjoint intervals $\left(I_{n}^{k}\right)$ and a sequence of mutually disjoint intervals $\left(J_{n}^{k}\right)$ such that $\sum_{n=1}^{\infty} l\left(I_{n}^{k}\right)$ and $\sum_{n=1}^{\infty} l\left(J_{n}^{k}\right)$ converge in $\mathcal{R}, \cup_{n=1}^{\infty} I_{n}^{k} \subset$ $A_{k} \subset \cup_{n=1}^{\infty} J_{n}^{k}$, and $\sum_{n=1}^{\infty} l\left(J_{n}^{k}\right)-\sum_{n=1}^{\infty} l\left(I_{n}^{k}\right) \leq d^{k} \epsilon$. Since $\lim _{k \rightarrow \infty} m\left(A_{k}\right)=0$ and since $\sum_{n=1}^{\infty} l\left(I_{n}^{k}\right) \leq \sum_{n=1}^{\infty} l\left(J_{n}^{k}\right) \leq m\left(A_{k}\right)+d^{k} \epsilon$, we obtain that

$$
\lim _{k \rightarrow \infty} \sum_{n=1}^{\infty} l\left(I_{n}^{k}\right)=\lim _{k \rightarrow \infty} \sum_{n=1}^{\infty} l\left(J_{n}^{k}\right)=0 .
$$

Thus, we can write $\cup_{k=1}^{\infty} \cup_{n=1}^{\infty} J_{n}^{k}$ and $\cup_{k=1}^{\infty} \cup_{n=1}^{\infty} I_{n}^{k}$ as unions of mutually disjoint intervals, say $\cup_{i=1}^{\infty} J_{i}$ and $\cup_{i=1}^{\infty} I_{i}$, such that $\cup_{i=1}^{\infty} I_{i} \subset \cup_{k=1}^{\infty} A_{k} \subset \cup_{i=1}^{\infty} J_{i}, \sum_{i=1}^{\infty} l\left(J_{i}\right)$ and $\sum_{i=1}^{\infty} l\left(I_{i}\right)$ converge and

$$
\begin{aligned}
\sum_{i=1}^{\infty} l\left(J_{i}\right)-\sum_{i=1}^{\infty} l\left(I_{i}\right) & \leq \sum_{k=1}^{\infty}\left(\sum_{n=1}^{\infty} l\left(J_{n}^{k}\right)-\sum_{n=1}^{\infty} l\left(I_{n}^{k}\right)\right) \\
& \leq \sum_{k=1}^{\infty} d^{k} \epsilon=\frac{d}{1-d} \epsilon<\epsilon .
\end{aligned}
$$


This shows that $\cup_{k=1}^{\infty} A_{k}$ is measurable.

Since $\cup_{k=1}^{\infty} A_{k} \subset \cup_{i=1}^{\infty} J_{i}=\cup_{k=1}^{\infty} \cup_{n=1}^{\infty} J_{n}^{k}$, we obtain that

$$
\begin{aligned}
m\left(\cup_{k=1}^{\infty} A_{k}\right) & \leq m\left(\cup_{k=1}^{\infty} \cup_{n=1}^{\infty} J_{n}^{k}\right) \leq \sum_{k=1}^{\infty} \sum_{n=1}^{\infty} l\left(J_{n}^{k}\right) \\
& \leq \sum_{k=1}^{\infty}\left(m\left(A_{k}\right)+d^{k} \epsilon\right)<\sum_{k=1}^{\infty} m\left(A_{k}\right)+\epsilon .
\end{aligned}
$$

This is true for all $\epsilon>0$ in $\mathcal{R}$; and hence

$$
m\left(\cup_{k=1}^{\infty} A_{k}\right) \leq \sum_{k=1}^{\infty} m\left(A_{k}\right) .
$$

Now assume that the measurable sets $A_{k}$ are mutually disjoint, and let $\epsilon>0$ in $\mathcal{R}$ be given. Then there exists $K \in \mathbb{N}$ such that $\sum_{k>K} m\left(A_{k}\right)<\epsilon / 2$. Also by Proposition 2.2, since $\cup_{k=1}^{\infty} A_{k}$ is measurable, there exists a sequence of mutually disjoint intervals $\left(J_{n}\right)$ such that $\cup_{k=1}^{\infty} A_{k} \subset \cup_{n=1}^{\infty} J_{n}, \sum_{n=1}^{\infty} l\left(J_{n}\right)$ converges, and $\sum_{n=1}^{\infty} l\left(J_{n}\right)<m\left(\cup_{k=1}^{\infty} A_{k}\right)+\epsilon / 2$. Since $A_{k} \subset \cup_{i=1}^{\infty} A_{i} \subset \cup_{n=1}^{\infty} J_{n}$ for all $k \in\{1,2, \ldots, K\}$, and since $A_{1}, A_{2}, \ldots, A_{K}$ are mutually disjoint, it follows that for each $k \in\{1,2, \ldots, K\}$, there exists a sequence of mutually disjoint intervals $\left(J_{n}^{k}\right)$ such that $\sum_{n=1}^{\infty} l\left(J_{n}^{k}\right)$ converges in $\mathcal{R}, A_{k} \subset \cup_{n=1}^{\infty} J_{n}^{k} \subset \cup_{n=1}^{\infty} J_{n}$, and $\cup_{n=1}^{\infty} J_{n}^{1}, \cup_{n=1}^{\infty} J_{n}^{2}, \ldots, \cup_{n=1}^{\infty} J_{n}^{K}$ are mutually disjoint. Thus,

$$
\begin{aligned}
\sum_{k=1}^{K} m\left(A_{k}\right) & \leq \sum_{k=1}^{K} m\left(\cup_{n=1}^{\infty} J_{n}^{k}\right)=\sum_{k=1}^{K} \sum_{n=1}^{\infty} l\left(J_{n}^{k}\right) \\
& \leq \sum_{n=1}^{\infty} l\left(J_{n}\right)<m\left(\cup_{k=1}^{\infty} A_{k}\right)+\frac{\epsilon}{2} .
\end{aligned}
$$

Hence

$$
\begin{aligned}
\sum_{k=1}^{\infty} m\left(A_{k}\right) & =\sum_{k=1}^{K} m\left(A_{k}\right)+\sum_{k>K} m\left(A_{k}\right) \\
& <m\left(\cup_{k=1}^{\infty} A_{k}\right)+\frac{\epsilon}{2}+\frac{\epsilon}{2}=m\left(\cup_{k=1}^{\infty} A_{k}\right)+\epsilon .
\end{aligned}
$$

This is true for all $\epsilon>0$ in $\mathcal{R}$; and hence

$$
\sum_{k=1}^{\infty} m\left(A_{k}\right) \leq m\left(\cup_{k=1}^{\infty} A_{k}\right)
$$

Combining the results of Equation (2.4) and Equation (2.5), we finally obtain that $m\left(\cup_{k=1}^{\infty} A_{k}\right)=\sum_{k=1}^{\infty} m\left(A_{k}\right)$, as claimed.

Proposition 2.7. Let $K \in \mathbb{N}$ be given and for each $k \in\{1, \ldots, K\}$, let $A_{k}$ be measurable. Then $\cap_{k=1}^{K} A_{k}$ is measurable and $m\left(\cap_{k=1}^{K} A_{k}\right) \leq \min _{1 \leq k \leq K} m\left(A_{k}\right)$.

Proof. Using induction on $K$, it suffices to show that if $A$ and $B$ are measurable sets in $\mathcal{R}$ then so is $A \cap B$, and $m(A \cap B) \leq \min \{m(A), m(B)\}$. So let $A, B \subset$ $\mathcal{R}$ be measurable and let $\epsilon>0$ in $\mathcal{R}$ be given. Then there exist sequences of mutually disjoint intervals $\left(I_{n}^{1}\right),\left(I_{n}^{2}\right),\left(J_{n}^{1}\right),\left(J_{n}^{2}\right)$ such that $\cup_{n=1}^{\infty} I_{n}^{1} \subset A \subset \cup_{n=1}^{\infty} J_{n}^{1}$, $\cup_{n=1}^{\infty} I_{n}^{2} \subset B \subset \cup_{n=1}^{\infty} J_{n}^{2}, \sum_{n=1}^{\infty} l\left(I_{n}^{1}\right), \sum_{n=1}^{\infty} l\left(I_{n}^{2}\right), \sum_{n=1}^{\infty} l\left(J_{n}^{1}\right), \sum_{n=1}^{\infty} l\left(J_{n}^{2}\right)$ all converge in $\mathcal{R}, \sum_{n=1}^{\infty} l\left(J_{n}^{1}\right)-\sum_{n=1}^{\infty} l\left(I_{n}^{1}\right) \leq \epsilon$ and $\sum_{n=1}^{\infty} l\left(J_{n}^{2}\right)-\sum_{n=1}^{\infty} l\left(I_{n}^{2}\right) \leq \epsilon$. 
It follows that $\left(\cup_{n=1}^{\infty} I_{n}^{1}\right) \cap\left(\cup_{n=1}^{\infty} I_{n}^{2}\right)$ forms a sequence of mutually disjoint intervals, say $\cup_{n=1}^{\infty} I_{n}$, such that $\sum_{n=1}^{\infty} l\left(I_{n}\right)$ converges in $\mathcal{R}$ and $\cup_{n=1}^{\infty} I_{n} \subset A \cap B$. Similarly, $\left(\cup_{n=1}^{\infty} J_{n}^{1}\right) \cap\left(\cup_{n=1}^{\infty} J_{n}^{2}\right)$ forms a sequence of mutually disjoint intervals, say $\cup_{n=1}^{\infty} J_{n}$, such that $\sum_{n=1}^{\infty} l\left(J_{n}\right)$ converges in $\mathcal{R}$ and $A \cap B \subset \cup_{n=1}^{\infty} J_{n}$. Since $\cup_{n=1}^{\infty} I_{n} \subset \cup_{n=1}^{\infty} I_{n}^{1}$ and $\cup_{n=1}^{\infty} J_{n} \subset \cup_{n=1}^{\infty} J_{n}^{1}$, and since each of the four sequences of intervals consists of mutually disjoint intervals, we obtain that

$$
\sum_{n=1}^{\infty} l\left(J_{n}\right)-\sum_{n=1}^{\infty} l\left(I_{n}\right) \leq \sum_{n=1}^{\infty} l\left(J_{n}^{1}\right)-\sum_{n=1}^{\infty} l\left(I_{n}^{1}\right) \leq \epsilon .
$$

Thus, $\cup_{n=1}^{\infty} I_{n} \subset A \cap B \subset \cup_{n=1}^{\infty} J_{n}$ and $\sum_{n=1}^{\infty} l\left(J_{n}\right)-\sum_{n=1}^{\infty} l\left(I_{n}\right) \leq \epsilon$. Hence $A \cap B$ is measurable.

Since $A \cap B \subset A$, we have that $m(A \cap B) \leq m(A)$. Similarly, $m(A \cap B) \leq m(B)$. Thus, $m(A \cap B) \leq \min \{m(A), m(B)\}$.

Proposition 2.8. Let $A, B \subset \mathcal{R}$ be measurable. Then $m(A \cup B)=m(A)+m(B)-$ $m(A \cap B)$.

Proof. First we note that, by Proposition 2.6 and Proposition 2.7, $A \cup B$ and $A \cap B$ are measurable. We show that $m(A)+m(B)=m(A \cup B)+m(A \cap B)$. Let $\epsilon>0$ in $\mathcal{R}$ be given. Then, using Proposition 2.2, there exists a sequence of mutually disjoint intervals $\left(J_{n}\right)$ such that $A \cup B \subset \cup_{n=1}^{\infty} J_{n}, \sum_{n=1}^{\infty} l\left(J_{n}\right)$ converges in $\mathcal{R}$ and $\sum_{n=1}^{\infty} l\left(J_{n}\right) \leq m(A \cup B)+\epsilon / 2$.

Since $A \backslash(A \cap B), B \backslash(A \cap B)$, and $A \cap B$ are mutually disjoint subsets of $A \cup B$, it follows that $\cup_{n=1}^{\infty} J_{n}$ can be rewritten as the disjoint union of three unions of mutually disjoint intervals, say $\cup_{n=1}^{\infty} J_{n}^{1}, \cup_{n=1}^{\infty} J_{n}^{2}$, and $\cup_{n=1}^{\infty} J_{n}^{3}$, such that

$$
A \backslash(A \cap B) \subset \cup_{n=1}^{\infty} J_{n}^{1}, B \backslash(A \cap B) \subset \cup_{n=1}^{\infty} J_{n}^{2}, A \cap B \subset \cup_{n=1}^{\infty} J_{n}^{3},
$$

and $\sum_{n=1}^{\infty} l\left(J_{n}^{1}\right), \sum_{n=1}^{\infty} l\left(J_{n}^{2}\right)$, and $\sum_{n=1}^{\infty} l\left(J_{n}^{3}\right)$ converge in $\mathcal{R}$. From

$$
A=A \backslash(A \cap B) \cup(A \cap B) \subset\left(\cup_{n=1}^{\infty} J_{n}^{1}\right) \cup\left(\cup_{n=1}^{\infty} J_{n}^{3}\right)
$$

we obtain that

$$
m(A) \leq m\left(\cup_{n=1}^{\infty} J_{n}^{1} \cup \cup_{n=1}^{\infty} J_{n}^{3}\right)=\sum_{n=1}^{\infty} l\left(J_{n}^{1}\right)+\sum_{n=1}^{\infty} l\left(J_{n}^{3}\right) .
$$

Also, from $B=B \backslash(A \cap B) \cup(A \cap B) \subset\left(\cup_{n=1}^{\infty} J_{n}^{2}\right) \cup\left(\cup_{n=1}^{\infty} J_{n}^{3}\right)$, we have that

$$
m(B) \leq m\left(\cup_{n=1}^{\infty} J_{n}^{2} \cup \cup_{n=1}^{\infty} J_{n}^{3}\right)=\sum_{n=1}^{\infty} l\left(J_{n}^{2}\right)+\sum_{n=1}^{\infty} l\left(J_{n}^{3}\right)
$$

Hence

$$
\begin{aligned}
m(A)+m(B) & \leq \sum_{n=1}^{\infty} l\left(J_{n}^{1}\right)+\sum_{n=1}^{\infty} l\left(J_{n}^{2}\right)+2 \sum_{n=1}^{\infty} l\left(J_{n}^{3}\right) \\
& =\sum_{n=1}^{\infty} l\left(J_{n}\right)+\sum_{n=1}^{\infty} l\left(J_{n}^{3}\right) \\
& \leq m(A \cup B)+\frac{\epsilon}{2}+m(A \cap B)+\frac{\epsilon}{2} \\
& =m(A \cup B)+m(A \cap B)+\epsilon
\end{aligned}
$$


where we have used the fact that

$$
\sum_{n=1}^{\infty} l\left(J_{n}^{3}\right)-m(A \cap B) \leq \sum_{n=1}^{\infty} l\left(J_{n}\right)-m(A \cup B) \leq \frac{\epsilon}{2} .
$$

Thus, $m(A)+m(B) \leq m(A \cup B)+m(A \cap B)+\epsilon$ for all $\epsilon>0$ in $\mathcal{R}$; and hence

$$
m(A)+m(B) \leq m(A \cup B)+m(A \cap B) .
$$

Now we prove the other inequality. Let $\epsilon>0$ in $\mathcal{R}$ be given. Then, again by Proposition 2.2, there exist a sequence of mutually disjoint intervals $\left(J_{n}^{1}\right)$ and a sequence of mutually disjoint intervals $\left(J_{n}^{2}\right)$ such that $A \subset \cup_{n=1}^{\infty} J_{n}^{1}, B \subset \cup_{n=1}^{\infty} J_{n}^{2}$, $\sum_{n=1}^{\infty} l\left(J_{n}^{1}\right)$ and $\sum_{n=1}^{\infty} l\left(J_{n}^{2}\right)$ converge, and

$$
\sum_{n=1}^{\infty} l\left(J_{n}^{1}\right) \leq m(A)+\frac{\epsilon}{2} \text { and } \sum_{n=1}^{\infty} l\left(J_{n}^{2}\right) \leq m(B)+\frac{\epsilon}{2} .
$$

Since $B \backslash(A \cap B)$ and $A \cap B$ are mutually disjoint subsets of $B$, it follows that we can rewrite $\cup_{n=1}^{\infty} J_{n}^{2}$ as the disjoint union of two unions of mutually disjoint intervals, say $\cup_{n=1}^{\infty} J_{n}^{2,1}$ and $\cup_{n=1}^{\infty} J_{n}^{2,2}$, such that

$$
B \backslash(A \cap B) \subset \cup_{n=1}^{\infty} J_{n}^{2,1}, A \cap B \subset \cup_{n=1}^{\infty} J_{n}^{2,2},
$$

and $\sum_{n=1}^{\infty} l\left(J_{n}^{2,1}\right)$ and $\sum_{n=1}^{\infty} l\left(J_{n}^{2,2}\right)$ converge in $\mathcal{R}$. From

$$
A \cup B=A \cup(B \backslash(A \cap B)) \subset\left(\cup_{n=1}^{\infty} J_{n}^{1}\right) \cup\left(\cup_{n=1}^{\infty} J_{n}^{2,1}\right),
$$

we obtain that

$$
\begin{aligned}
m(A \cup B) & \leq m\left(\cup_{n=1}^{\infty} J_{n}^{1} \cup \cup_{n=1}^{\infty} J_{n}^{2,1}\right)=\sum_{n=1}^{\infty} l\left(J_{n}^{1}\right)+\sum_{n=1}^{\infty} l\left(J_{n}^{2,1}\right) \\
& \leq m(A)+\frac{\epsilon}{2}+\sum_{n=1}^{\infty} l\left(J_{n}^{2,1}\right) .
\end{aligned}
$$

Also, since $A \cap B \subset \cup_{n=1}^{\infty} J_{n}^{2,2}$, we obtain that $m(A \cap B) \leq \sum_{n=1}^{\infty} l\left(J_{n}^{2,2}\right)$. Hence

$$
\begin{aligned}
m(A \cup B)+m(A \cap B) & \leq m(A)+\frac{\epsilon}{2}+\sum_{n=1}^{\infty} l\left(J_{n}^{2,1}\right)+\sum_{n=1}^{\infty} l\left(J_{n}^{2,2}\right) \\
& =m(A)+\frac{\epsilon}{2}+\sum_{n=1}^{\infty} l\left(J_{n}^{2}\right) \\
& \leq m(A)+\frac{\epsilon}{2}+m(B)+\frac{\epsilon}{2} \\
& =m(A)+m(B)+\epsilon .
\end{aligned}
$$

This is true for all $\epsilon>0$ in $\mathcal{R}$; and hence

$$
m(A \cup B)+m(A \cap B) \leq m(A)+m(B) \text {. }
$$

Combining the results of Equation (2.6) and Equation (2.7), we obtain that $m(A \cup B)+m(A \cap B)=m(A)+m(B)$ or $m(A \cup B)=m(A)+m(B)-m(A \cap B)$.

Finally, we note that the complement of a measurable set in a measurable set need not be measurable. For example, $[0,1]$ and $[0,1] \cap \mathbb{Q}$ are both measurable with measures 1 and 0 , respectively. However, the complement of $[0,1] \cap \mathbb{Q}$ in $[0,1]$ is not measurable. On the other hand, if $B \subset A \subset \mathcal{R}$ and if $A, B$ and $A \backslash B$ are all measurable, then it follows from Proposition 2.6 that $m(A)=m(B)+m(A \backslash B)$. 
The example of $[0,1] \backslash[0,1] \cap \mathbb{Q}$ above shows that the axiom of choice is not needed here to construct a nonmeasurable set, as there are many simple examples of nonmeasurable sets. Indeed, any uncountable real subset of $\mathcal{R}$, like $[0,1] \cap \mathbb{R}$ for example, is not measurable.

\section{Measurable Functions}

Like in $\mathbb{R}$, we first introduce a family of simple functions on $\mathcal{R}$ from which we will obtain a larger family of measurable functions.

3.1. Simple Functions. In the Lebesgue measure theory on $\mathbb{R}$, the simple functions consist only of step functions (piece-wise constant functions); and all measurable functions including all monomials, polynomials and power series are obtained as uniform limits of simple functions. It can be easily shown that in $\mathcal{R}$ the order topology is too strong and none of the monomials can be obtained as a uniform limit of polynomials of lower degrees. So using the step functions as our simple functions would yield a too small class of functions that we can integrate. So we introduce a larger family of simple functions. Here we define such a family of simple functions in an abstract way, which we will use throughout the discussions in this paper; and we will give two examples in Remark 4.15 at the end of the paper.

Definition 3.1. Let $a<b$ in $\mathcal{R}$ be given and $\mathcal{S}(a, b)$ a family of functions from $I(a, b)$ to $\mathcal{R}$. Then we say that $\mathcal{S}(a, b)$ is a family of simple functions on $I(a, b)$ if the following are true:

(1) $\mathcal{S}(a, b)$ is an algebra that contains the identity function;

(2) for all $f \in \mathcal{S}(a, b), f$ is Lipschitz on $I(a, b)$ and there exists an anti-derivative $F$ of $f$ in $\mathcal{S}(a, b)$;

(3) for all differentiable $f \in \mathcal{S}(a, b)$, if $f^{\prime}=0$ on $I(a, b)$ then $f$ is constant on $I(a, b)$; moreover, if $f^{\prime} \leq 0$ on $I(a, b)$ then $f$ is nonincreasing on $I(a, b)$.

If $f \in \mathcal{S}(a, b)$, we say that $f$ is simple on $I(a, b)$.

It follows from the first condition in Definition 3.1 that any constant function on $I(a, b)$ is in $\mathcal{S}(a, b)$; moreover, if $f, g \in \mathcal{S}(a, b)$ and if $\alpha \in \mathcal{R}$, then $f+\alpha g \in \mathcal{S}(a, b)$. Also, it follows from the third condition that the anti-derivative in the second condition is unique up to a constant. A close look at Definition 3.1 reveals that the polynomials algebra on $I(a, b)$ is the smallest family of simple functions on $I(a, b)$. Two examples of larger families of simple functions are discussed at the end of the paper, in Remark 4.15.

While the third condition in Definition 3.1 is automatically satisfied in real analysis, this is not the case in $\mathcal{R}$, as the following example shows.

Example 3.2. Let $g:(0,1) \rightarrow \mathcal{R}$ be given by $g(x)[q]=x[q / 3]$ for all $q \in \mathbb{Q}$. Then $g$ is differentiable on $(0,1)$ with $g^{\prime}(x)=0$ for all $x \in(0,1)$. We first observe that $g(x+y)=g(x)+g(y)$ for all $x, y \in(0,1)$. Now let $x \in(0,1)$ and $\epsilon>0$ in $\mathcal{R}$ be given. Let $\delta=\min \{\epsilon, d\}$, and let $y \in(0,1)$ be such that $0<|y-x|<\delta$. Then

$$
\left|\frac{g(y)-g(x)}{y-x}\right|=\left|\frac{g(y-x)}{y-x}\right| \sim(y-x)^{2} \text { since } g(y-x) \sim(y-x)^{3} .
$$

Since $|y-x|<\min \{\epsilon, d\}$, we obtain that $(y-x)^{2} \ll \epsilon$. Hence

$$
\left|\frac{g(y)-g(x)}{y-x}\right|<\epsilon \text { for all } y \in(0,1) \text { satisfying } 0<|y-x|<\delta \text {; }
$$


which shows that $g$ is differentiable at $x$ and $g^{\prime}(x)=0$.

Now let $f:(0,1) \rightarrow \mathcal{R}$ be given by $f(x)=g(x)-x^{4}$. Then $f$ is differentiable on $(0,1)$ with $f^{\prime}(x)=-4 x^{3}<0$ for all $x \in(0,1)$. However, we have that $d>d^{2}$ and $f(d)=d^{3}-d^{4}>f\left(d^{2}\right)=d^{6}-d^{8}$. Thus, even though $f^{\prime}<0$ everywhere on $(0,1)$, $f$ is not nonincreasing on $(0,1)$.

As we will see in Section 3.2, starting with the family of simple functions, we will be able to obtain a larger family of measurable functions. Then, in Section 4, by just requiring the integral to satisfy one fundamental property, we show that there is only one way to define the integral of a simple function over an interval. Then, based on that, we show how to integrate any measurable function over a measurable set.

\subsection{Measurable Functions.}

Definition 3.3. Let $A \subset \mathcal{R}$ be a measurable subset of $\mathcal{R}$ and let $f: A \rightarrow \mathcal{R}$ be bounded on $A$. Then we say that $f$ is measurable on $A$ if for all $\epsilon>0$ in $\mathcal{R}$, there exists a sequence of mutually disjoint intervals $\left(I_{n}\right)$ such that $I_{n} \subset A$ for all $n$, $\sum_{n=1}^{\infty} l\left(I_{n}\right)$ converges in $\mathcal{R}, m(A)-\sum_{n=1}^{\infty} l\left(I_{n}\right) \leq \epsilon$ and $f$ is simple on $I_{n}$ for all $n$.

Proposition 3.4 (Characterization of Measurable Functions). Let $A \subset \mathcal{R}$ be measurable and let $f: A \rightarrow \mathcal{R}$ be measurable. Then $f$ is locally a simple function almost everywhere on $A$.

Proof. Let $A_{1}=\{x \in A: f$ is not locally simple around $x\}$. We show that $A_{1}$ is measurable and $m\left(A_{1}\right)=0$. Let $\epsilon>0$ in $\mathcal{R}$ be given. Then, since $A$ is measurable and since $f$ is measurable on $A$, there exist a sequence of mutually disjoint open intervals $\left(a_{n}, b_{n}\right)$ and a sequence of mutually disjoint intervals $\left(J_{n}\right)$ such that $\cup_{n=1}^{\infty}\left(a_{n}, b_{n}\right) \subset A \subset \cup_{n=1}^{\infty} J_{n}, f$ is simple on $\left(a_{n}, b_{n}\right)$ for each $n \in \mathbb{N}$, $\sum_{n=1}^{\infty}\left(b_{n}-a_{n}\right)$ and $\sum_{n=1}^{\infty} l\left(J_{n}\right)$ converge, $m(A)-\sum_{n=1}^{\infty}\left(b_{n}-a_{n}\right) \leq \epsilon / 2$ and $\sum_{n=1}^{\infty} l\left(J_{n}\right)-m(A) \leq \epsilon / 2$. It follows that

$$
A_{1} \subset A \backslash \cup_{n=1}^{\infty}\left(a_{n}, b_{n}\right) \subset \cup_{n=1}^{\infty} J_{n} \backslash \cup_{n=1}^{\infty}\left(a_{n}, b_{n}\right),
$$

where $\cup_{n=1}^{\infty} J_{n} \backslash \cup_{n=1}^{\infty}\left(a_{n}, b_{n}\right)$ can be written as a union of mutually disjoint intervals, say $\cup_{n=1}^{\infty} J_{n}^{1}$, such that $\sum_{n=1}^{\infty} l\left(J_{n}^{1}\right)$ converges in $\mathcal{R}$ and $\sum_{n=1}^{\infty} l\left(J_{n}^{1}\right)=\sum_{n=1}^{\infty} l\left(J_{n}\right)-$ $\sum_{n=1}^{\infty}\left(b_{n}-a_{n}\right)$. For each $n \in \mathbb{N}$, let $I_{n}^{1}=\emptyset$. Then $\cup_{n=1}^{\infty} I_{n}^{1} \subset A_{1} \subset \cup_{n=1}^{\infty} J_{n}^{1}$ and

$$
\begin{aligned}
\sum_{n=1}^{\infty} l\left(J_{n}^{1}\right)-\sum_{n=1}^{\infty}\left(I_{n}^{1}\right) & =\sum_{n=1}^{\infty} l\left(J_{n}^{1}\right)=\sum_{n=1}^{\infty} l\left(J_{n}\right)-\sum_{n=1}^{\infty}\left(b_{n}-a_{n}\right) \\
& =\left(\sum_{n=1}^{\infty} l\left(J_{n}\right)-m(A)\right)+\left(m(A)-\sum_{n=1}^{\infty}\left(b_{n}-a_{n}\right)\right) \\
& \leq \frac{\epsilon}{2}+\frac{\epsilon}{2}=\epsilon .
\end{aligned}
$$

Thus, $A_{1}$ is measurable. Moreover, since $A_{1} \subset \cup_{n=1}^{\infty} J_{n}^{1}$, we obtain that $m\left(A_{1}\right) \leq$ $\sum_{n=1}^{\infty} l\left(J_{n}^{1}\right) \leq \epsilon$. This is true for all $\epsilon>0$ in $\mathcal{R}$; and hence $m\left(A_{1}\right)=0$.

The following example shows that the converse of Proposition 3.4 need not be true.

Example 3.5. Let $f:[0,1] \rightarrow \mathcal{R}$ be given by

$$
f(x)= \begin{cases}0 & \text { if } x \in[0,1] \cap \mathbb{Q} \\ x & \text { otherwise }\end{cases}
$$


Then $f$ is locally simple almost everywhere on $[0,1]$; but $f$ is not measurable on $[0,1]$.

As an immediate result of Proposition 3.4 and the properties of simple functions, we obtain the following result which will prove very useful in defining the integral of a measurable function, as we will see in details in Section 4.

Corollary 3.6. Let $a<b$ in $\mathcal{R}$ and let $f: I(a, b) \rightarrow \mathcal{R}$ be measurable. Then $f$ is continuous almost everywhere on $I(a, b)$. Moreover, if $f$ is differentiable on $I(a, b)$ and if $f^{\prime}$ vanishes everywhere, then $f$ is constant on $I(a, b)$.

Proof. The first part follows directly from Proposition 3.4 and the fact that a simple function is continuous everywhere on its domain.

Now assume that $f$ is differentiable on $I(a, b)$ and that $f^{\prime}(x)=0$ for all $x \in$ $I(a, b)$. Then it follows from Proposition 3.4 that $f$ is locally constant almost everywhere on $I(a, b)$; this is so since a simple function has a vanishing derivative on a whole interval if and only if it is constant on that interval [11]. Thus, $f$ is locally constant on $I(a, b)$ except on a discrete set of points in $I(a, b)$; and this, together with the fact that $f$ is differentiable on $I(a, b)$, entails that $f$ is constant on the whole interval $I(a, b)$.

Corollary 3.7. Let $a<b$ in $\mathcal{R}$ and let $f, g:[a, b] \rightarrow \mathcal{R}$ be measurable. Assume that $f$ and $g$ are both differentiable with $f^{\prime}=g^{\prime}$ on $[a, b]$. Then there exists a constant $c$ such that $f(x)=g(x)+c$ for all $x \in[a, b]$; and hence $f(b)-f(a)=g(b)-g(a)$.

Proposition 3.8. Let $A, B \subset \mathcal{R}$ be measurable, let $f$ be a measurable function on $A$ and $B$. Then $f$ is measurable on $A \cup B$ and $A \cap B$.

Proof. Let $\epsilon>0$ in $\mathcal{R}$ be given. Then, since $f$ is measurable on $A$, there exists a sequence of mutually disjoint intervals $\left(I_{n}^{1}\right)$ such that $\cup_{n=1}^{\infty} I_{n}^{1} \subset A, \sum_{n=1}^{\infty} l\left(I_{n}^{1}\right)$ converges, $m(A)-\sum_{n=1}^{\infty} l\left(I_{n}^{1}\right) \leq \epsilon / 2$, and $f$ is simple on $I_{n}^{1}$ for all $n \geq 1$. Also, since $f$ is measurable on $B$, there exists a sequence of mutually disjoint intervals $\left(I_{n}^{2}\right)$ such that $\cup_{n=1}^{\infty} I_{n}^{2} \subset B, \sum_{n=1}^{\infty} l\left(I_{n}^{2}\right)$ converges, $m(B)-\sum_{n=1}^{\infty} l\left(I_{n}^{2}\right) \leq \epsilon / 2$, and $f$ is simple on $I_{n}^{2}$ for all $n \geq 1$.

It follows that $\left(\cup_{n=1}^{\infty} I_{n}^{1}\right) \cup\left(\cup_{n=1}^{\infty} I_{n}^{2}\right)$ can be written as a union of mutually disjoint intervals, say $\cup_{n=1}^{\infty} I_{n}$, such that $\cup_{n=1}^{\infty} I_{n}=\left(\cup_{n=1}^{\infty} I_{n}^{1}\right) \cup\left(\cup_{n=1}^{\infty} I_{n}^{2}\right) \subset A \cup B$, $\sum_{n=1}^{\infty} l\left(I_{n}\right)$ converges, and $f$ is simple on $I_{n}$ for all $n \geq 1$. Moreover,

$$
m(A \cup B)-\sum_{n=1}^{\infty} l\left(I_{n}\right) \leq m(A)-\sum_{n=1}^{\infty} l\left(I_{n}^{1}\right)+m(B)-\sum_{n=1}^{\infty} l\left(I_{n}^{2}\right) \leq \frac{\epsilon}{2}+\frac{\epsilon}{2}=\epsilon
$$

This shows that $f$ is measurable on $A \cup B$.

Also, $\left(\cup_{n=1}^{\infty} I_{n}^{1}\right) \cap\left(\cup_{n=1}^{\infty} I_{n}^{2}\right)$ can be written as a union of mutually disjoint intervals, say $\cup_{n=1}^{\infty} I_{n}^{0}$, such that $\cup_{n=1}^{\infty} I_{n}^{0}=\left(\cup_{n=1}^{\infty} I_{n}^{1}\right) \cap\left(\cup_{n=1}^{\infty} I_{n}^{2}\right) \subset A \cap B, \sum_{n=1}^{\infty} l\left(I_{n}^{0}\right)$ converges, and $f$ is simple on $I_{n}^{0}$ for all $n \geq 1$. Moreover, using Proposition 2.8, we 
have that

$$
\begin{aligned}
m(A \cap B)-\sum_{n=1}^{\infty} l\left(I_{n}^{0}\right)= & m(A)+m(B)-m(A \cup B) \\
& +\sum_{n=1}^{\infty} l\left(I_{n}\right)-\sum_{n=1}^{\infty} l\left(I_{n}^{1}\right)-\sum_{n=1}^{\infty} l\left(I_{n}^{2}\right) \\
\leq & m(A)+m(B)-\sum_{n=1}^{\infty} l\left(I_{n}^{1}\right)-\sum_{n=1}^{\infty} l\left(I_{n}^{2}\right) \\
& =m(A)-\sum_{n=1}^{\infty} l\left(I_{n}^{1}\right)+m(B)-\sum_{n=1}^{\infty} l\left(I_{n}^{2}\right) \\
\leq & \epsilon .
\end{aligned}
$$

Thus, $f$ is measurable on $A \cap B$.

Proposition 3.9. Let $A \subset \mathcal{R}$ be measurable, let $f, g: A \rightarrow \mathcal{R}$ be measurable and let $\alpha \in \mathcal{R}$ be given. Then $f+\alpha g$ and $f \cdot g$ are measurable on $A$.

Proof. Let $\epsilon>0$ in $\mathcal{R}$ be given. Then there exist a sequence of mutually disjoint intervals $\left(I_{n}^{1}\right)$ and a sequence of mutually disjoint intervals $\left(I_{n}^{2}\right)$ such that $\cup_{n=1}^{\infty} I_{n}^{1} \subset$ $A, \cup_{n=1}^{\infty} I_{n}^{2} \subset A, \sum_{n=1}^{\infty} l\left(I_{n}^{1}\right)$ and $\sum_{n=1}^{\infty} l\left(I_{n}^{2}\right)$ converge, $m(A)-\sum_{n=1}^{\infty} l\left(I_{n}^{1}\right) \leq \epsilon / 2$, $m(A)-\sum_{n=1}^{\infty} l\left(I_{n}^{2}\right) \leq \epsilon / 2, f$ is simple on $I_{n}^{1}$ for all $n \in \mathbb{N}$ and $g$ is simple on $I_{n}^{2}$ for all $n \in \mathbb{N}$.

It follows that $\left(\cup_{n=1}^{\infty} I_{n}^{1}\right) \cap\left(\cup_{n=1}^{\infty} I_{n}^{2}\right)$ can be written as a union of mutually disjoint intervals, say $\cup_{n=1}^{\infty} I_{n}^{0}$, such that $\cup_{n=1}^{\infty} I_{n}^{0}=\left(\cup_{n=1}^{\infty} I_{n}^{1}\right) \cap\left(\cup_{n=1}^{\infty} I_{n}^{2}\right) \subset A, \sum_{n=1}^{\infty} l\left(I_{n}^{0}\right)$ converges, and $f+\alpha g$ and $f \cdot g$ are simple on $I_{n}^{0}$ for all $n \geq 1$ (where we use the fact that the sum and product of simple functions are again simple). Moreover, again using Proposition 2.8, we have that

$$
\begin{aligned}
m(A)-\sum_{n=1}^{\infty} l\left(I_{n}^{0}\right) & =m(A)+m\left(\cup_{n=1}^{\infty} I_{n}^{1} \cup \cup_{n=1}^{\infty} I_{n}^{2}\right)-\sum_{n=1}^{\infty} l\left(I_{n}^{1}\right)-\sum_{n=1}^{\infty} l\left(I_{n}^{2}\right) \\
& \leq m(A)+m(A)-\sum_{n=1}^{\infty} l\left(I_{n}^{1}\right)-\sum_{n=1}^{\infty} l\left(I_{n}^{2}\right) \\
& =m(A)-\sum_{n=1}^{\infty} l\left(I_{n}^{1}\right)+m(A)-\sum_{n=1}^{\infty} l\left(I_{n}^{2}\right) \\
& \leq \frac{\epsilon}{2}+\frac{\epsilon}{2}=\epsilon .
\end{aligned}
$$

Thus, $f+\alpha g$ and $f \cdot g$ are measurable on $A$.

The following example shows that the uniform limit of measurable functions need not be measurable.

Example 3.10. For each $k \in \mathbb{N}$, let $f_{k}:[0,1] \rightarrow \mathcal{R}$ be given by $f_{k}(x)=d^{j}$ if $x$ belongs to one of the open intervals deleted from $[0,1]$ at the $j$ th step of the construction of the real Cantor set $C$ for $1 \leq j \leq k$, and $f_{k}(x)=d^{k+1}$ if $x$ belongs to the closed set left from $[0,1]$ at the $k$ th step of the construction of $C$. Then the sequence $\left(f_{k}\right)$ converges uniformly on $[0,1]$; but the limit function is not measurable on $[0,1]$. The proofs of the last two statements are left as an exercise for the reader. 


\section{INTEGRATION}

After having introduced measurable functions and studied their properties in Section 3, we show in this section how to find the measure or integral of a given measurable function $f$ over a measurable set $A$, which we will denote by $\int_{A} f$. However we define the integral, we would like it to satisfy as many of the basic properties of real integrals as possible. One such fundamental property of real integrals is that the integral of the derivative of a differentiable function over an interval is equal to the difference between the function values at the endpoints. By requiring just that, namely that

$$
\int_{[a, b]} f^{\prime}=f(b)-f(a)
$$

for a differentiable measurable function $f$ on $[a, b]$ (which is a well-defined requirement by Corollary 3.7), then we will show that there is only one way to define the integral of any measurable function $f$ over any measurable set $A \subset \mathcal{R}$.

First assume that $f: I(a, b) \rightarrow \mathcal{R}$ is simple and let $F$ be a simple anti-derivative of $f$ on $I(a, b)$. Then $F$ is measurable and differentiable on $I(a, b)$. If $I(a, b)$ is closed, we use Equation (4.1) and define the integral of $f$ over $I(a, b)=[a, b]$ as $F(b)-F(a)$. If $I(a, b)$ is not closed, then we can extend $F$ to a new simple function $\bar{F}:[a, b] \rightarrow \mathcal{R}$, such that

$$
\vec{F}(x)=\left\{\begin{array}{ll}
F(x) & \text { if } x \in(a, b) \\
\lim _{x \rightarrow a} F(x) & \text { if } x=a \\
\lim _{x \rightarrow b} F(x) & \text { if } x=b
\end{array} .\right.
$$

Then $\bar{F}$ is an anti-derivative of $\bar{f}$, the extension of $f$ on $[a, b]$. So it is natural in this case to require that $\int_{I(a, b)} f=\int_{[a, b]} \bar{f}$; which leads to

$$
\int_{I(a, b)} f=\bar{F}(b)-\bar{F}(a)=\lim _{x \rightarrow b} F(x)-\lim _{x \rightarrow a} F(x) .
$$

That the limits in defining the extensions exist follow from the fact that the simple functions are Lipschitz. We combine the two cases above (closed interval or otherwise) into one expression and define the integral of a simple function over an interval.

Definition 4.1. Let $a<b$ in $\mathcal{R}$, let $f: I(a, b) \rightarrow \mathcal{R}$ be simple on $I(a, b)$, and let $F$ be a simple anti-derivative of $f$ on $I(a, b)$. Then the integral of $f$ over $I(a, b)$ is the $\mathcal{R}$ number

$$
\int_{I(a, b)} f=\lim _{x \rightarrow b} F(x)-\lim _{x \rightarrow a} F(x) .
$$

The following result is an immediate consequence of Definition 4.1.

Proposition 4.2. Let $a<b$ in $\mathcal{R}$ and let $\alpha \in \mathcal{R}$ be a given constant. Then $\int_{I(a, b)} \alpha=\alpha(b-a)$.

Proof. First we note that $\alpha x$ is a simple anti-derivative of the constant function $\alpha$ on $I(a, b)$. Thus, $\int_{I(a, b)} \alpha=\lim _{x \rightarrow b}(\alpha x)-\lim _{x \rightarrow a}(\alpha x)=\alpha(b-a)$.

Proposition 4.3. Let $a<b$ in $\mathcal{R}$, let $f, g: I(a, b) \rightarrow \mathcal{R}$ be simple, and let $\alpha \in \mathcal{R}$ be given. Then $\int_{I(a, b)}(f+\alpha g)=\int_{I(a, b)} f+\alpha \int_{I(a, b)} g$. 
Proof. Let $F$ and $G$ be simple anti-derivatives of $f$ and $g$ on $I(a, b)$, respectively. Then $F+\alpha G$ is an anti-derivative of $f+\alpha g$ on $I(a, b)$. Thus,

$$
\begin{aligned}
\int_{I(a, b)}(f+\alpha g) & =\lim _{x \rightarrow b}(F+\alpha G)(x)-\lim _{x \rightarrow a}(F+\alpha G)(x) \\
& =\lim _{x \rightarrow b}(F(x)+\alpha G(x))-\lim _{x \rightarrow a}(F(x)+\alpha G(x)) \\
& =\left(\lim _{x \rightarrow b} F(x)-\lim _{x \rightarrow a} F(x)\right)+\alpha\left(\lim _{x \rightarrow b} G(x)-\lim _{x \rightarrow a} G(x)\right) \\
& =\int_{I(a, b)} f+\alpha \int_{I(a, b)} g .
\end{aligned}
$$

Proposition 4.4. Let $a<b$ in $\mathcal{R}$, let $f: I(a, b) \rightarrow \mathcal{R}$ be simple and nonpositive on $I(a, b)$. Then $\int_{I(a, b)} f \leq 0$.

Proof. Let $F$ be a simple anti-derivative of $f$ on $I(a, b)$. Then $\int_{I(a, b)} f=\lim _{x \rightarrow b} F(x)-$ $\lim _{x \rightarrow a} F(x)$. Since $F^{\prime}=f \leq 0$ on $I(a, b), F$ is nonincreasing on $I(a, b)$. It follows that $\lim _{x \rightarrow b} F(x) \leq \lim _{x \rightarrow a} F(x)$; and hence $\int_{I(a, b)} f \leq 0$.

Using Proposition 4.3 and applying Proposition 4.4 to $f-g$, we readily obtain:

Corollary 4.5. Let $a<b$ in $\mathcal{R}$, let $f, g: I(a, b) \rightarrow \mathcal{R}$ be simple and satisfy $f \leq g$ on $I(a, b)$. Then $\int_{I(a, b)} f \leq \int_{I(a, b)} g$.

Corollary 4.6. Let $a<b$ in $\mathcal{R}$, let $f: I(a, b) \rightarrow \mathcal{R}$ be simple on $I(a, b)$ and let $M$ be $a$ bound of $|f|$ on $I(a, b)$. Then

$$
\left|\int_{I(a, b)} f\right| \leq M(b-a) .
$$

Proof. Using Corollary 4.5 and the fact that $f \leq|f| \leq M$ on $I(a, b)$, we obtain that

$$
\int_{I(a, b)} f \leq \int_{I(a, b)} M=M(b-a) .
$$

Also noting that $-f \leq|f| \leq M$ on $I(a, b)$, we obtain that

$$
-\int_{I(a, b)} f=\int_{I(a, b)}(-f) \leq M(b-a) .
$$

Combining the results of Equation (4.2) and Equation (4.3), we finally obtain the desired result.

Now let $A \subset \mathcal{R}$ be measurable, let $f: A \rightarrow \mathcal{R}$ be measurable and let $M$ be a bound for $|f|$ on $A$. Then for every $k \in \mathbb{N}$, there exists a sequence of mutually disjoint intervals $\left(I_{n}^{k}\right)_{n \in \mathbb{N}}$ such that $\cup_{n=1}^{\infty} I_{n}^{k} \subset A, \sum_{n=1}^{\infty} l\left(I_{n}^{k}\right)$ converges, $m(A)-$ $\sum_{n=1}^{\infty} l\left(I_{n}^{k}\right) \leq d^{k}$, and $f$ is simple on $I_{n}^{k}$ for all $n \in \mathbb{N}$. Without loss of generality, we may assume that $I_{n}^{k} \subset I_{n}^{k+1}$ for all $n \in \mathbb{N}$ and for all $k \in \mathbb{N}$. Since $\lim _{n \rightarrow \infty} l\left(I_{n}^{k}\right)=0$, and since $\left|\int_{I_{n}^{k}} f\right| \leq M l\left(I_{n}^{k}\right)$ by Corollary 4.6, it follows that

$$
\lim _{n \rightarrow \infty} \int_{I_{n}^{k}} f=0 \text { for all } k \in \mathbb{N} \text {. }
$$


Thus, $\sum_{n=1}^{\infty} \int_{I_{n}^{k}} f$ converges in $\mathcal{R}$ for all $k \in \mathbb{N}[11]$.

Next we show that the sequence $\left(\sum_{n=1}^{\infty} \int_{I_{n}^{k}} f\right)_{k \in \mathbb{N}}$ converges in $\mathcal{R}$. So let $\epsilon>0$ be given in $\mathcal{R}$; and let $K \in \mathbb{N}$ be such that $M d^{K} \leq \epsilon$. Let $k>j \geq K$ be given in $\mathbb{N}$. Then $\cup_{n=1}^{\infty} I_{n}^{k} \backslash \cup_{n=1}^{\infty} I_{n}^{j}$ can be written as a union of mutually disjoint intervals, say $\left(I_{n}^{j, k}\right)_{n \in \mathrm{N}}$, such that $\sum_{n=1}^{\infty} l\left(I_{n}^{j, k}\right)$ converges, and

$$
\sum_{n=1}^{\infty} l\left(I_{n}^{j, k}\right)=\sum_{n=1}^{\infty} l\left(I_{n}^{k}\right)-\sum_{n=1}^{\infty} l\left(I_{n}^{j}\right) \leq m(A)-\sum_{n=1}^{\infty} l\left(I_{n}^{j}\right) \leq d^{j} \leq d^{K} .
$$

Thus,

$$
\begin{aligned}
\left|\sum_{n=1}^{\infty} \int_{I_{n}^{k}} f-\sum_{n=1}^{\infty} \int_{I_{n}^{j}} f\right| & =\left|\sum_{n=1}^{\infty} \int_{I_{n}^{j, k}} f\right| \leq \sum_{n=1}^{\infty}\left|\int_{I_{n}^{j, k}} f\right| \\
& \leq \sum_{n=1}^{\infty} M l\left(I_{n}^{j, k}\right)=M \sum_{n=1}^{\infty} l\left(I_{n}^{j, k}\right) \\
& \leq M d^{K} \leq \epsilon,
\end{aligned}
$$

where we have used the fact that an infinite series converges if and only if it converges absolutely [11]. Thus, the sequence $\left(\sum_{n=1}^{\infty} \int_{I_{n}^{k}} f\right)_{k \in \mathbb{N}}$ is Cauchy; and hence it converges in $\mathcal{R}$. We define the unique limit as the integral of $f$ over $A$.

Definition 4.7. Let $A \subset \mathcal{R}$ be measurable and let $f: A \rightarrow \mathcal{R}$ be measurable. Then the integral of $f$ over $A$, denoted by $\int_{A} f$, is given by

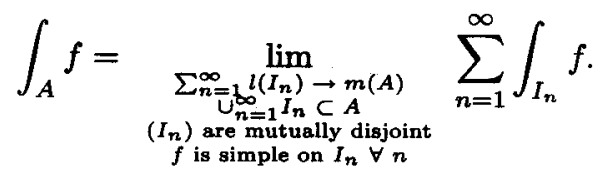

Proposition 4.8. Let $A \subset \mathcal{R}$ be measurable and let $\alpha \in \mathcal{R}$ be given. Then $\int_{A} \alpha=$ $\alpha m(A)$.

Proof. Using Definition 4.7, we have that

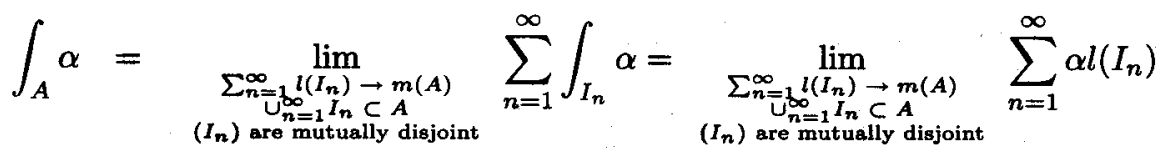

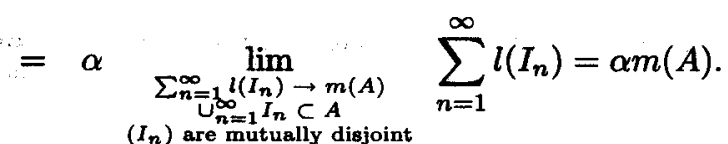

In particular, we have that $m(A)=\int_{A} 1$.

Proposition 4.9. Let $A \subset \mathcal{R}$ be measurable and let $f: A \rightarrow \mathcal{R}$ be measurable and nonpositive on $A$. Then $\int_{A} f \leq 0$.

Proof. Let $\epsilon>0$ in $\mathcal{R}$ be given. Then there exists a sequence of mutually disjoint intervals $\left(I_{n}\right)$ such that $\cup_{n=1}^{\infty} I_{n} \subset A, \sum_{n=1}^{\infty} l\left(I_{n}\right)$ converges, $m(A)-\sum_{n=1}^{\infty} l\left(I_{n}\right) \leq$ $\epsilon$, and $f$ is simple on $I_{n}$ for all $n \in \mathbb{N}$. It follows, using Proposition 4.4, that $\sum_{n=1}^{\infty} \int_{I_{n}} f \leq 0$; and hence $\int_{A} f=\lim _{\epsilon \rightarrow 0} \sum_{n=1}^{\infty} \int_{I_{n}} f \leq 0$. 
Using the proof of Proposition 3.9 and a limiting argument similar to that in the proof of Proposition 4.9, we obtain:

Proposition 4.10. Let $A \subset \mathcal{R}$ be measurable, let $f, g: A \rightarrow \mathcal{R}$ be measurable, and let $\alpha \in \mathcal{R}$ be given. Then $\int_{A}(f+\alpha g)=\int_{A} f+\alpha \int_{A} g$.

As a consequence of Propositions 4.9 and 4.10, we obtain the following result.

Corollary 4.11. Let $A \subset \mathcal{R}$ be measurable and let $f, g: A \rightarrow \mathcal{R}$ be measurable and satisfy $f \leq g$ on $A$. Then $\int_{A} f \leq \int_{A} g$.

Corollary 4.12. Let $A \subset \mathcal{R}$ be measurable, let $f: A \rightarrow \mathcal{R}$ be measurable and let $M$ be a bound for $|f|$ on $A$. Then $\left|\int_{A} f\right| \leq M m(A)$.

Using the proof of Proposition 3.8, and using the limiting argument in the proof of Proposition 4.9, we obtain the following result.

Proposition 4.13. Let $A, B \subset \mathcal{R}$ be measurable and let $f$ be a measurable function on $A$ and $B$. Then

$$
\int_{A \cup B} f=\int_{A} f+\int_{B} f-\int_{A \cap B} f .
$$

Theorem 4.14. Let $A \subset \mathcal{R}$ be measurable, let $f: A \rightarrow \mathcal{R}$, for each $k \in \mathbb{N}$ let $f_{k}: A \rightarrow \mathcal{R}$ be measurable on $A$, and let the sequence $\left(f_{k}\right)$ converge uniformly to $f$ on $A$. Then $\lim _{k \rightarrow \infty} \int_{A} f_{k}$ exists. Moreover, if $f$ is measurable on $A$, then $\lim _{k \rightarrow \infty} \int_{A} f_{k}=\int_{A} f$.

Proof. Let $\epsilon>0$ in $\mathcal{R}$ be given and let

$$
\epsilon_{1}=\left\{\begin{array}{ll}
\epsilon / m(A) & \text { if } m(A) \neq 0 \\
\epsilon & \text { if } m(A)=0
\end{array} .\right.
$$

Then $\epsilon_{1}>0$ and there exists $K \in \mathbb{N}$ such that $\left|f_{k}(x)-f_{j}(x)\right| \leq \epsilon_{1}$ for all $k, j \geq K$ and for all $x \in A$. It follows that

$$
\left|\int_{A} f_{k}-\int_{A} f_{j}\right|=\left|\int_{A}\left(f_{k}-f_{j}\right)\right| \leq \epsilon_{1} m(A) \leq \epsilon \text { for all } k, j \geq K .
$$

Thus, the sequence $\left(\int_{A} f_{k}\right)$ is Cauchy. Since $\mathcal{R}$ is Cauchy complete in the order topology, the sequence $\left(\int_{A} f_{k}\right)$ converges in $\mathcal{R}$; that is, $\lim _{k \rightarrow \infty} \int_{A} f_{k}$ exists in $\mathcal{R}$.

Now assume that $f$ is measurable on $A$; to show that $\lim _{k \rightarrow \infty} \int_{A} f_{k}=\int_{A} f$, we follow the same steps as in the first part of the proof, replacing $f_{j}$ by $f$.

Remark 4.15. Power series $[11,12]$ are one example of a family of simple functions on any interval in their domain of convergence. Prior to $[11,12]$, work on power series on the Levi-Civita field has been restricted to power series with real coefficients. In $[5,6,7,4]$, they were studied for infinitely small arguments, while in [1], using the weak topology, also finite arguments were possible. In [11], the general case of $\mathcal{R}$ coefficients and arguments is considered. A radius of convergence $\eta$ is derived such that the power series converges weakly for all points whose distance from the center is finitely smaller than $\eta$ and it converges strongly (in the order topology) for all points whose distance from the center is infinitely smaller than $\eta$.

In [12] it is shown that within their radius of convergence, power series are infinitely often differentiable and the derivatives to any order are obtained by differentiating the power series term by term. Also, power series can be re-expanded around any point in their domain of convergence and the radius of convergence of the new 
series is equal to the difference between the radius of convergence of the original series and the distance between the original and new centers of the series. Furthermore, it is shown in $[11,12]$ that power series satisfy all the common theorems of real calculus on a closed interval of $\mathcal{R}$, like the intermediate value theorem, the maximum theorem and the mean value theorem and that they satisfy the criteria for a family of simple functions on any interval in their domain of convergence.

Research currently in progress aims at generalizing the results in $[11,12]$ to power series with rational exponents. We show that unless the coefficients in the series form a null sequence in the order topology, the sequence of exponents must be left-finite for the series to have a positive radius of convergence. In the latter case, we derive a radius of convergence which depends on the density of the exponents and on the coefficients in the series.

We then show that within their domain of convergence, generalized power series with rational exponents have similar properties to those of regular power series. In particular, they satisfy the intermediate value theorem and the mean value theorem and they can be re-expanded around any point in their domain of convergence. We also show that the generalized power series with rational exponents satisfy the criteria for a family of simple functions on any interval in their domain of convergence.

\section{REFERENCES}

[1] M. Berz. Calculus and Numerics on Levi-Civita Fields. In M. Berz, C. Bischof, G. Corliss, and A. Griewank, editors, Computational Differentiation: Techniques, Applications, and Tools, pages 19-35, Philadelphia, 1996. SIAM.

[2] M. Berz. Analytical and Computational Methods for the Levi-Civita Fields. In Lecture Notes in Pure and Applied Mathematics, pages 21-34. Marcel Dekker, Proceedings of the Sixth International Conference on P-adic Analysis, July 2-9, 2000, ISBN 0-8247-0611-0.

[3] Wolfgang Krull. Allgemeine Bewertungstheorie. J. Reine Angew. Math., 167:160-196, 1932.

[4] D. Laugwitz. Tullio Levi-Civita's work on nonarchimedean structures (with an Appendix: Properties of Levi-Civita fields). In Atti Dei Convegni Lincei 8: Convegno Internazionale Celebrativo Del Centenario Della Nascita De Tullio Levi-Civita, Academia Nazionale dei Lincei, Roma, 1975.

[5] Tullio Levi-Civita. Sugli infiniti ed infinitesimi attuali quali elementi analitici. Atti Ist. Veneto di Sc., Lett. ed Art., 7a, 4:1765, 1892.

[6] Tullio Levi-Civita. Sui numeri transfiniti. Rend. Acc. Lincei, 5a, 7:91,113, 1898.

[7] L. Neder. Modell einer Leibnizschen Differentialrechnung mit aktual unendich kleinen Größen. Mathematische Annalen, 118:718-732, 1941-1943.

[8] S. Priess-Crampe. Angeordnete Strukturen: Gruppen, Körper, projektive Ebenen. Springer, Berlin, 1983.

[9] Paulo Ribenboim. Fields: Algebraically Closed and Others. Manuscripta Mathematica, 75:115-150, 1992.

[10] K. Shamseddine. New Elements of Analysis on the Levi-Civita Field. PhD thesis, Michigan State University, East Lansing, Michigan, USA, 1999. also Michigan State University report MSUCL-1147.

[11] K. Shamseddine and M. Berz. Convergence on the Levi-Civita Field and Study of Power Series. In Lecture Notes in Pure and Applied Mathematics, pages 283-299. Marcel Dekker, Proceedings of the Sixth International Conference on P-adic Analysis, July 2-9, 2000, ISBN 0-8247-0611-0.

[12] K. Shamseddine and M. Berz. Analytical Properties of Power Series on Non-Archimedean Fields. submitted. see also Michigan State University report MSUCL-1163. 
Department of Mathematics and Department of Physics and Astronomy, Michigan State University, EAST LANSING, MI 48824

E-mail address: khodr@math.msu.edu

Department of Physics and Astronomy, Michigan State University, East Lansing, Mi 48824

E-mail address: berz@msu. edu 